\title{
Differential Phase Delay Estimation in VRAD Mission of SELENE (KAGUYA)
}

\author{
By Fuyuhiko KIKUCHI ${ }^{1)}$, Qinghui LIU $^{2)}$, Natalia PETROVA ${ }^{3)}$, Yuji HARADA ${ }^{1)}$, Hideo HANADA ${ }^{1)}$, \\ Takahiro IWATA $^{4)}$, Noriyuki NAMIKI ${ }^{5)}$, Nobuyuki KAWANO ${ }^{2)}$, and Sho SASAKI ${ }^{1)}$ \\ 1) RISE Project, National Astronomical Observatory, Oshu, Japan \\ ${ }^{2}$ Shanghai Astronomical Observatory, Shanghai, China \\ ${ }^{3)}$ Kazan State University, Kazan, Russian Federation \\ ${ }^{4)}$ The Institute of Space and Astronautical Science, Sagamihara, Japan \\ ${ }^{5)}$ Planetary Exploration Research Center, The Chiba Institute of Technology, Narashino, Japan
}

(Received May 2nd, 2008)

\begin{abstract}
In the very long baseline interferometry (VLBI) radio sources mission of selenological and engineering explorer, the differential phase delay between the Rstar and Vstar sub-satellites is obtained by using the multifrequency VLBI method during the switching VLBI observation period. The cycle ambiguity is successfully determined and the differential phase delay is estimated within an error of 7 picoseconds. The RMS error is somewhat larger than that for the case of same-beam VLBI because fluctuations of propagation delays whose periods are shorter than the switching interval cannot be canceled out between Rstar and Vstar. However, the differential phase delay during the switching VLBI period is sufficiently accurate and, together with Doppler and range measurements, can be a useful means for precisely determining satellite orbits and precisely estimating the lunar gravity field.
\end{abstract}

Key Words: Same-beam VLBI, Switching VLBI, Cycle Ambiguity, Phase Delay, VRAD

\section{Introduction}

The moon's gravity field is a key point in enabling human beings to ascertain the moon's origin and evolution. However, lack of accurate information about the moon's gravity field, especially for its far side and limb region, has restricted attempts to accurately determine the moon's moment of inertia ${ }^{1,2)}$. To improve the accuracy with which the lunar gravity field can be determined, especially for the moon's limb region, a differential very long baseline interferometry (VLBI) observation mission was carried out in the differential VLBI RADio sources (VRAD) of the Japanese lunar explorer SELenological and ENgineering Explorer (SELENE/KAGUYA) ${ }^{2)}$.

Measurements of differential phase delay (DPD), which is a doubly differenced delay between the Rstar and Vstar sub-satellites of SELENE, are sensitive to the relative position and velocity of $\mathrm{R} / \mathrm{Vstar}$ in the direction perpendicular to the line-of-sight (LOS). On the other hand, Doppler and range measurements are sensitive to the LOS direction. Therefore, R/Vstar's three-dimensional motion and the lunar gravity field can be determined more precisely by combining these measurements. Our simulation results indicate that combining these measurements enables the low degree coefficients of the lunar gravity field to be derived more accurately, i.e., by more than one order of magnitude, than has been possible with previous methods ${ }^{3)}$. The DPD of R/Vstar can be successfully estimated during same-beam VLBI observation periods ${ }^{4)}$. In this case, the root mean square
(RMS) error of the DPD in a 60-second integration interval is about 2 picoseconds (ps) for the four Japanese baselines. This result confirms that the desired accuracy of VRAD, which is 3.3 ps in a 110 -second integration interval, can be achieved $^{2)}$. However, the same-beam VLBI observation period accounts for about $60 \%$ of the total observation period in VRAD ${ }^{5)}$. In order to estimate the lunar gravity field at the level of accuracy desired for VRAD, the DPD in the switching VLBI observation period is an important factor. Although it is difficult to estimate the DPD under cloudy conditions and/or when traveling ionospheric disturbance occurs in the ionosphere above the VLBI station ${ }^{5)}$, there are cases in which the DPD can be successfully estimated. This paper describes the method we used to estimate the DPD during the switching VLBI observation period and shows the results we obtained. It also briefly describes the VRAD system, the VLBI network, the switching and the same-beam VLBI observation method.

\section{VRAD System}

\subsection{On-board system}

The VLBI radio sources RAD1 and RAD2 are loaded on R/Vstar ${ }^{2)}$. The three S-band (S1, S2, and S3) and one $\mathrm{X}$-band $(\mathrm{X} 1)$ radio signals are transmitted from $\mathrm{RAD} 1 / 2$ (fs $1=2212[\mathrm{MHz}], \mathrm{fs} 2=2218[\mathrm{MHz}], \mathrm{fs} 3=2287[\mathrm{MHz}]$ ), $\mathrm{fx} 1=8456[\mathrm{MHz}])$. Only the X-band signal from Rstar is a noise signal $(100-\mathrm{kHz}$ bandwidth) and the others are carrier wave signals. 


\subsection{Ground system}

The narrow bandwidth VLBI back-end system is used for VRAD ${ }^{6}$. The system is composed of a phase calibrator, a low-pass filter, and a low-rate sampling and recording system called the "S-RTP station". The S-RTP station's sampling rate is $200 \mathrm{ksps}$ and it has six quantization bits. The three S-band and one X-band signals are recorded separately in the four channels of S-RTP station.

\subsection{Data processing}

Original software constructed for the VRAD mission was used for data processing ${ }^{6)}$. The software comprised a correlation module and a delay estimation module, which corresponds to the software used in the multi-frequency VLBI (MFV) method. The correlation process was carried out by FX mode. The FX type correlator first Fourier transforms the frequency domain and then does a multiplication operation. In the course of calculating the residual fringe phase (RFP) from the cross-correlation function, only several Fourier components, which correspond to the carrier frequency and its vicinity, are picked up. Because the bandwidth of the signal from $\mathrm{R} /$ Vstar is very narrow (i.e., less than $10 \mathrm{~Hz}$ ), it is possible to reduce noise and obtain a high signal-to-noise ratio.

\subsection{VLBI network}

The VLBI network of VRAD consists of four domestic Japanese VLBI Exploration of Radio Astrometry (VERA) stations: MIZUSAWA, OGASAWARA, ISHIGAKI, and IRIKI. Observations were carried out over this network thrice a week continuously over a nine-month period. After the nominal mission was completed, an extended mission was carried out. The four Japanese stations worked together with four foreign stations, i.e., SHANGHAI and URUMQI (China), HOBART (Australia), and WETTZELL (Germany), in conducting two intensive one-month observation periods in January 2008 and May 2008 .

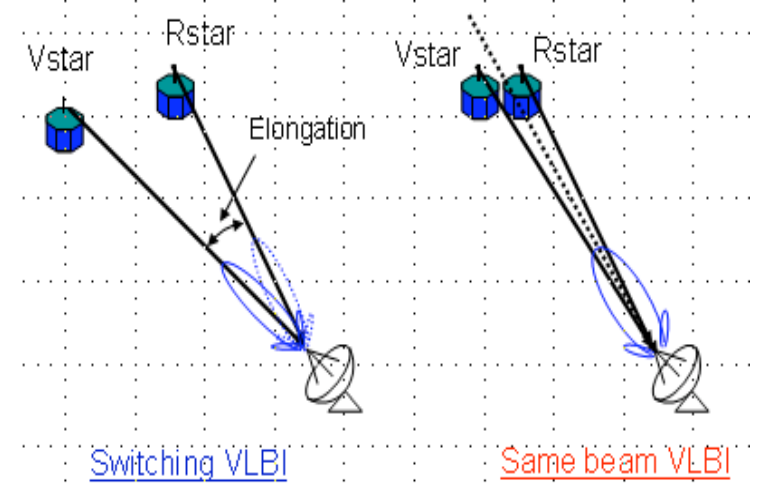

Fig. 1. Switching and same-beam VLBI observation methods.

\section{Application of Switching and Same-beam VLBI Observation to MFV Method}

The phase delay method carried out by means of the multi-frequency VLBI (MFV) method has been proposed for VRAD by evolving the conventional group delay $\operatorname{method}^{7)}$. With this method, four different frequency signals whose frequencies are allocated to resolve the cycle ambiguity are used in VRAD. In order to determine the unique value of the cycle ambiguity, three conditions for the MFV method must be satisfied. First, the RMS error of the differential residual fringe phase (DRFP) of the signals from R/Vstar, $\sigma_{\phi \mathrm{s}}$, and $\sigma_{\phi \mathrm{x}}$ must be less than 4.3 degrees in the S-band and 179 degrees in the X-band signals. Second, the doubly differenced total electron content (TEC) of the ionosphere through which four propagation paths from the two spacecraft to two ground stations $\triangle^{2} \mathrm{D}$ must be canceled out or compensated within an error of 0.23 TEC unit (TECU, 1 TECU is $10^{16} \mathrm{el} / \mathrm{m}^{2}$ ). Third, the a priori delay $\Delta^{2} \tau$ a priori, which is needed for the correlation process, must be known within an error of 83 nanoseconds (ns).

In order to satisfy these conditions, two kinds of differential VLBI observation methods were carried out in VRAD. The first is the switching VLBI observation method. This method is suitable when the elongation between R/Vstar is larger than the beam width of the ground antenna (0.37 degrees and 0.1 degrees for S-band and X-band signals in the case of the VERA 20m antenna). By alternately observing R/Vstar with a switching interval of $T s w$, the tropospheric and the ionospheric fluctuations whose period is shorter than $T s w$ can be canceled out. The other method is the same-beam VLBI observation method $^{5,8)}$. With this method, when the elongation is smaller than the beam width of the ground antenna, the middle point of R/Vstar is tracked and its signals can be simultaneously received. In this case, it can be expected that most of the fluctuations will be canceled out.

\section{Observation Results for Switching VLBI Period}

\subsection{Connection of residual fringe phase}

Fig. 2 and Fig. 3 show the RFP of R/Vstar for each scan during the switching VLBI observation period. Scans $n$, $n+2, n+4, \ldots$ are for Rstar and scans $n+1, n+3, n+5, \ldots$ are for Vstar. For the switching VLBI case, the DRFP is calculated as follows: First, the RFP is connected such that there is no cycle ambiguity between scans as shown in Fig. 2 and Fig. 3. Second, the RFP between scans is interpolated from the polynomial fitting function, which is calculated from the RFP of each scan as shown in Fig. 4. Third, the RFP for each scan is integrated at the first epoch of each scan. Finally, the DRFP is calculated from the difference between the RFP of Rstar and Vstar.

\subsection{MFV method conditions}

Before estimating the cycle ambiguity from the DRFP, the conditions of the MFV method must be estimated. The conditions are shown in Table 1. Although the RMS error of the DRFP is larger than that for same-beam VLBI, the RMS error conditions can be satisfied for both the S- and $\mathrm{X}$-bands. The reason for the large RMS error is that the switching VLBI method cannot cancel out the fluctuation of a propagation delay whose period is shorter than the switching interval. The doubly differenced TEC is estimated from the DRFP of S/X-band signals as shown in Fig. $5^{5)}$. The RMS error is 0.01 TECU and TEC does not exceed +/- 0.23 TECU. The error of the a priori delay is 
less than 83 ns under the use of the orbit provided by Japan aerospace exploration agency.

\subsection{Estimation of differential delay}

Finally, the cycle ambiguity is uniquely estimated as shown in Fig. 6 and the differential group/phase delays are estimated by using the cycle ambiguity as shown in Fig. 7.

It is considered that the temporal trend shown in the differential delay is caused by the error of the a priori orbit of $\mathrm{R} / \mathrm{Vstar}$ and will disappear after the orbit is improved by precisely determining it through VLBI, Doppler, and range measurements. Therefore, the accuracy of the differential phase delay is evaluated from the RMS error after the temporal trend is removed by a 4th order polynomial fitting function. The RMS error of the differential phase delay of the $\mathrm{X}$-band signal in a 100 -second integration interval is $7 \mathrm{ps}$ for the IRIKI-OGASAWARA baseline. The bias of the DPD caused by the ionospheric delay is about 0.2 ps after the correction of the ionospheric delay. The RMS error is somewhat larger than that for the case of same-beam VLBI, which is about 2 ps in a 60 -second integration interval. This is because the fluctuation of the propagation delay whose period is shorter than the switching interval cannot be canceled out between R/Vstar.

\section{Conclusion}

We used both same-beam VLBI observation and switching VLBI observation to estimate differential phase delay of the Rstar and Vstar sub-satellites. This enabled us to successfully estimate the differential phase delay of $\mathrm{X}$-band signals without cycle ambiguity. Although the RMS error of 7 ps in a 100-second integration interval was somewhat larger than that for same-beam VLBI, it can be useful for further study. That is, accumulating VLBI delay data for both the same-beam and switching VLBI periods will enable us to carry out orbit determination and lunar gravity field estimation by using delay, 2-way and 4-way Doppler, and range data measurements.

\section{Acknowledgements}

The authors appreciate the contribution of all the engineers of NEC/Toshiba Space Systems Ltd. (NTS), Nippon Antenna Co. Ltd., and Nippi Corporation who diligently developed the onboard instruments and sub-satellites. We also express thanks to the entire staff of the Kaguya mission, and to Dr. Dirk Behrend and Dr. Yasuhiro Koyama of the international VLBI service committee for their support and encouragement. The gravity experiments conducted during the Kaguya mission would never have been achieved without the prominent expertise and profound knowledge of Mr. Fumio Fuke, an NTS engineer who passed away two months after the mission was launched. We express sincere thanks to him for his contributions to science and mourn his passing and the loss it entails for Japanese space development.

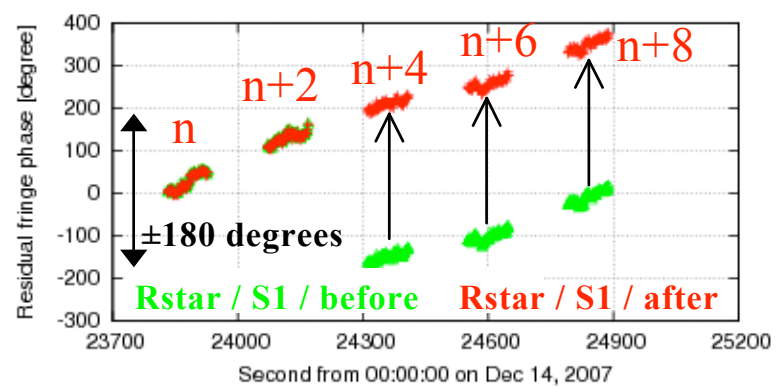

Fig. 2. Residual fringe phase of signal S1 from Rstar. Green points represent residual fringe before scans are connected and red points represent fringe for connected scans.

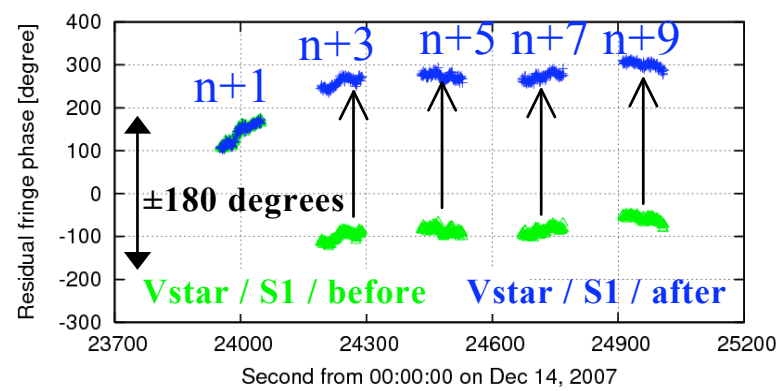

Fig. 3. Residual fringe phase of signal S1 from Vstar. Green points represent residual fringe before scans are connected and blue points represent fringe for connected scans.

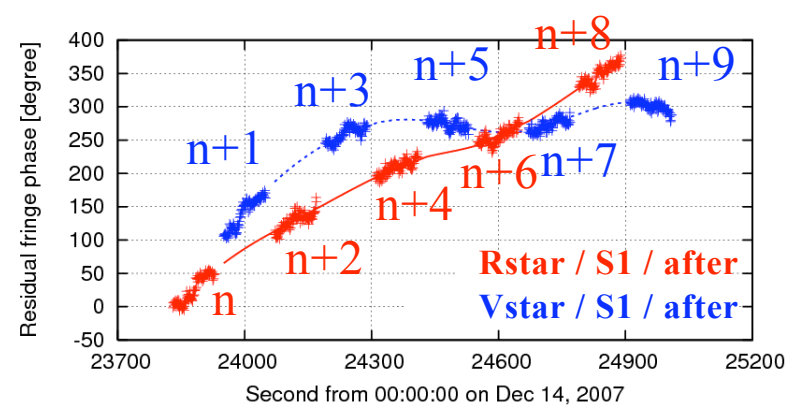

Fig. 4. Residual fringe phase of signal $\mathrm{S} 1$ from R/Vstar. Red and blue lines represent $4^{\text {th }}$ order polynomial fitting function of residual fringe phase from $\mathrm{R} / \mathrm{V}$ star.

Table 1. Estimated MFV method conditions.

\begin{tabular}{|c|c|c|c|c|}
\hline & $\begin{array}{l}\sigma_{\phi \mathrm{s}} \\
{[\text { degrees] }} \\
<4.3\end{array}$ & $\begin{array}{l}\triangle^{2} \mathrm{D} \\
{[\mathrm{TECU}]} \\
<0.23\end{array}$ & $\begin{array}{l}\Delta^{2} \tau_{\text {a priori }} \\
{[\mathrm{ns}]} \\
<83\end{array}$ & $\begin{array}{l}\sigma_{\phi x} \\
{[\text { degrees] }} \\
<179\end{array}$ \\
\hline $\mathrm{Nx} 1$ & 3.6 & 0.01 & $<83$ & 5.1 \\
\hline
\end{tabular}




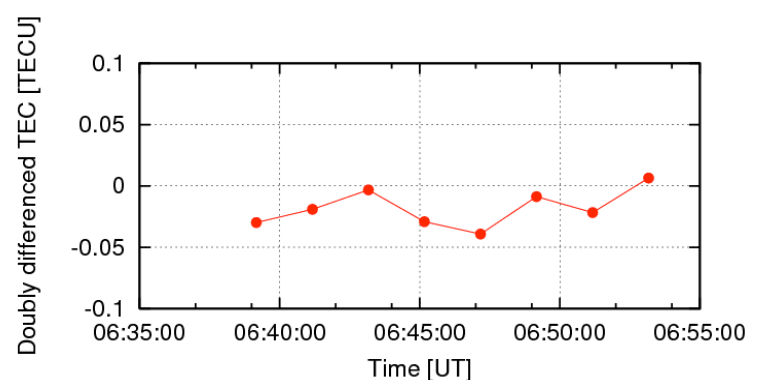

Fig. 5. Doubly differenced TEC estimated from DRFP of S/X-band signals.
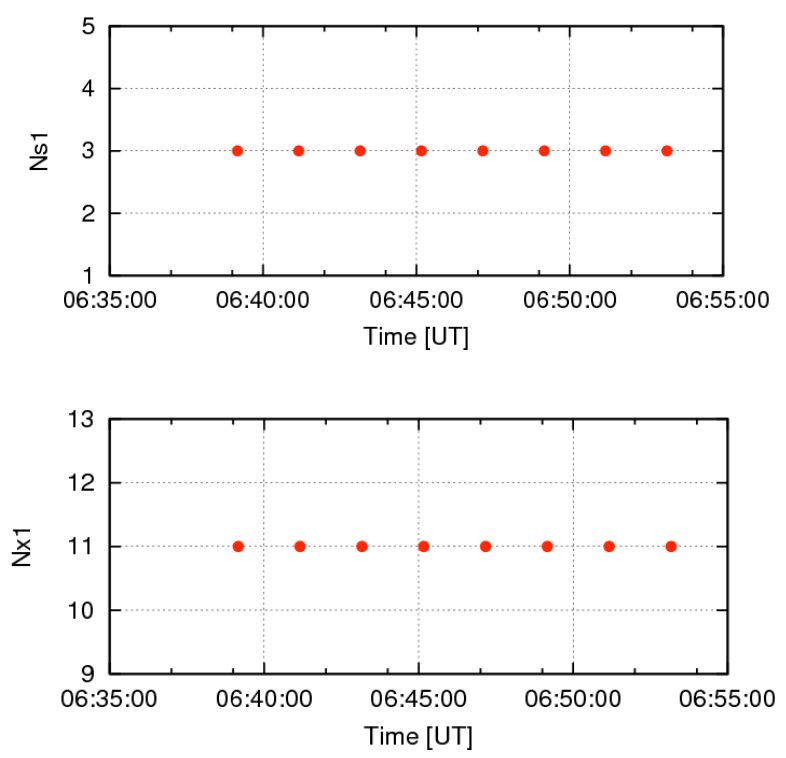

Fig. 6. Estimated cycle ambiguity of S-band signal S1 (upper) and $\mathrm{X}$-band signal X1 (lower).

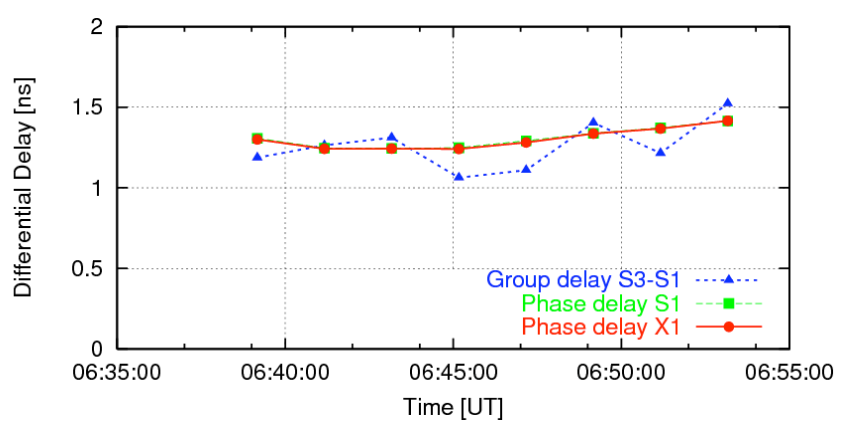

Fig. 7. Differential group delay of $\mathrm{S} 1$ and $\mathrm{S} 3$ and differential phase delay of S1 and X1.

\section{References}

1) Konopliv, A. S., S. W. Asmer, E. Carranza, W. L. Sjogren, and D. N. Yuan : Recent Gravity Models as a Result of the Lunar Prospector Mission, Icarus, 150 (2001), pp.1-8.

2) Hanada, H., T. Iwata, Y. Kono, and K. Matsumoto : VRAD Mission: Precise Observation of Orbits of Sub-satellites in SELENE, IVS 2002 General Meeting Proceedings, (2002), pp.73-76.

3) Matsumoto, K., H. Hanada, N. Namiki, T. Iwata, S. Goossens, S. Tsuruta, N. Kawano, and D. Rowlands : A simulation study for anticipated accuracy of lunar gravity field model by SELENE tracking data, Adv. Space Res., (2007), doi:10.1016/j.asr.2007.03.066 (in press).

4) Kikuchi, F., Q. Liu, N. Petrova, K. Matsumoto, Y. Ishihara, S. Goossens, K. Asari, S. Tsuruta, T. Ishikawa, H. Noda, H. Hanada, T. Iwata, N. Namiki, N. Kawano, and S. Sasaki : PRELIMINARY RESULTS FOR VRAD MISSION OF KAGUYA (SELENE), LPSC proceedings, (2008).

5) Kikuchi, F., Q. Liu, K. Matsumoto, H. Hanada, and N. Kawano : Simulation study of differential phase delay estimation by same beam VLBI method, Earth Planets Space, 60 (2008), pp.391-406.

6) Kikuchi, F., Y. Kono, M.Yoshikawa, M. Sekido, M. Ohnishi, Y. Murata, J.S. Ping, Q. Liu, K. Matsumoto, K. Asari, S. Tsuruta, H. Hanada, and N. Kawano : VLBI observation of narrow bandwidth signals from the spacecraft, Earth Planets Space, 56 (2004), pp.1041-1047.

7) Kono, Y., H. Hanada, J.S. Ping, Y. Koyama, Y. Fukuzaki, and N. Kawano : Precise positioning of spacecrafts by multi-frequency VLBI, Earth Planets Space, 55 (2003), pp.581-589.

8) Liu, Q., F. Kikuchi, K. Matsumoto, K. Asari, S. Tsuruta, J. Ping, H. Hanada, and N. Kawano : Error analysis of same-beam differential VLBI technique using two SELENE satellites, $A d v$. Space Res., 40 (2007), Issue 1, pp.51-57. 